\title{
Critical Role of Peripheral Actions of Intravenous Nicotine in Mediating Its Central Effects
}

\author{
Magalie Lenoir' and Eugene A Kiyatkin*,' \\ In-vivo Electrophysiology Unit, Behavioral Neuroscience Branch, National Institute on Drug Abuse-Intramural Research Program, \\ National Institutes of Health, Baltimore, MD, USA
}

\begin{abstract}
In addition to its direct action on central neurons, nicotine (NIC) activates multiple nicotinic acetylcholine receptors localized on afferent terminals of sensory nerves at the sites of its administration. Although the activation of these receptors is important in mediating the primary sensory and cardiovascular effects of $\mathrm{NIC}$, their role in triggering and maintaining the neural effects of $\mathrm{NIC}$ remains unclear. Using high-speed electroencephalography (EEG) and electromyography (EMG) recordings in freely moving rats, we showed that NIC at low intravenous (i.v.) doses $(10-30 \mu \mathrm{g} / \mathrm{kg}$ ) induced rapid, strong, and prolonged EEG desynchronization both in the cortex and ventral tegmental area (with decreases in $\alpha$ and robust increases in $\beta$ and $\gamma$ frequencies) and neck EMG activation that began during the injection ( $\sim 5 \mathrm{~s}$ ). EEG and EMG effects of NIC were drastically reduced by pre-treatment with hexamethonium, a peripherally acting NIC antagonist, and the immediate EEG effects of NIC were strongly inhibited during urethane anesthesia. Although NIC pyrrolidine methiodide, a quaternary NIC analog that cannot enter the brain, also induced rapid EEG desynchronization, its effects were much shorter and weaker than those of NIC. Therefore, NIC by acting on peripheral nicotinic receptors provides a major contribution to its rapid, excitatory effects following i.v. administration. Since this action creates a sensory signal that rapidly reaches the brain via neural pathways and precedes the slower and more prolonged direct actions of NIC on brain cells, it could have a major role in associative learning and changes in the behavioral and physiological effects of $\mathrm{NIC}$ following its repeated use.

Neuropsychopharmacology (201 I) 36, 2125-2138; doi:10.1038/npp.201 I.104; published online 8 June 201 I
\end{abstract}

Keywords: electroencephalography; electromyography; field potentials; peripheral nicotinic receptors; sensory signal; learning

\section{INTRODUCTION}

Nicotine (NIC) is considered the main active component of tobacco that makes smoking a highly addictive behavior. The actions of NIC are mediated by nicotinic acetylcholine $(\mathrm{nACh})$ receptors that are widely expressed both in the central and peripheral nervous systems (see Changeux, 2010; Gotti and Clementi, 2004, for review). Particularly, nACh receptors are present on afferents of visceral and somatic sensory nerves that densely innervate all locations of NIC administration (ie, lung alveoli, nasal and oral cavities, vessels) (Alimohammadi and Silver, 2000; Flores et al, 1996; Ginzel, 1975; Gu et al, 2008; Juan, 1982; MontiBloch and Eyzaguirre, 1980; Xu et al, 2007). While it is well established that the direct interaction of NIC with central neurons, particularly within the mesocorticolimbic

\footnotetext{
*Correspondence: Dr EA Kiyatkin, In-vivo Electrophysiology Unit, Behavioral Neuroscience Branch, National Institute on Drug AbuseIntramural Research Program, National Institutes of Health, DHHS, 333 Cassell Drive, Baltimore, MD 21224, USA, Tel: + I 4437402844 , Fax: + I 443740 2155, E-mail: ekiyatki@intra.nida.nih.gov

Received 2 March 20 I ; revised II April 20 I I; accepted I2 May 20 I I
}

dopamine system, is essential in mediating its reinforcing properties (see Balfour, 2009; Corrigall, 1991; Di Chiara, 2000 , for review), the role of peripheral actions of this drug remains more obscure.

Although NIC after smoking or intravenous (i.v.) delivery rapidly reaches the brain and easily crosses the blood-brain barrier (BBB; Berridge et al, 2010; Rose et al, 2010), its interaction with centrally located nACh receptors is always preceded by a transient activation of multiple afferents of sensory nerves at the sites of administration and within the circulatory system (Anand, 1996; Jonsson et al, 2002; Liu and Simon, 1996; Steen and Reeh, 1993; Walker et al, 1996). Therefore, in addition to the direct action on central neurons, NIC-induced changes in neural activity could occur due to changes in afferent input in response to peripherally driven sensory signal that rapidly arrives to the brain via visceral and somato-sensory pathways. Along with their role in acute sensory effects of NIC, peripheral nACh receptors appear to be also essential in mediating the major cardiovascular effects of i.v. NIC at low doses $(5-30 \mu \mathrm{g} / \mathrm{kg})$. These effects occur with the second-scale latencies, depend upon the site of drug administration, and are inhibited by peripherally acting NIC antagonists (Anand, 1996; Barlow 
and Dobson, 1955; Ginzel, 1975). Furthermore, stimulation of peripheral nACh receptors on sensory afferents has been implicated in mediating rapid, transient effects of i.v. NIC on central monoamine cells, particularly noradrenalinecontaining (Engberg and Hajos, 1994) and dopaminecontaining neurons (Erhardt et al, 2002).

In the present study, electroencephalography (EEG) and neck electromyography (EMG) with subsequent high-time resolution signal amplitude and frequency analyses were used in freely moving rats to examine rapid neural effects of i.v. NIC at low doses (10 and $30 \mu \mathrm{g} / \mathrm{kg}$ ) and explore basic mechanisms underlying these changes. While there are numerous studies aimed to correlate EEG signals with specific neuronal activity and neural processes (see Hobson, 1999, Buzsaki, 2006, Steriade and McCarley, 2005, for review), EEG desynchronization is a valuable index of global neural activation. EEG desynchronization can result from either sensory input, appear 'spontaneously' in association with awakening and behavioral activity or be induced by pharmacological drugs (Kiyatkin and Smirnov, 2010; McClung et al, 1976-1977; Sasaki et al, 1996). In addition, an event-related EEG desynchronization is typically tightly related to increased EMG activity that reflects alterations in tonic and phasic muscular activity, a centrally mediated peripheral response to the sensory signal. To explore the role of peripheral actions of NIC in mediating its neural effects, three strategies were employed. First, we tested the effects of i.v. NIC during blockade of peripheral nACh receptors induced by hexamethonium (HEXA), which cannot cross the BBB (Aceto et al, 1983; Gillis and Lewis, 1956). Since general anesthesia dramatically attenuates neural responses to somato-sensory stimuli and this sensory mechanism could be engaged in the processing of the NIC-induced peripheral neural signal, second, we examined how the effects of i.v. NIC are altered during urethane anesthesia. Third, we examined how the effects of NIC are mimicked by NIC pyrrolidine methiodide (NIC$\mathrm{PM}$ ), a quaternary BBB-impermeable NIC's analog. In addition to the cortex, electrical activity (or local field potentials) was recorded from the ventral tegmental area of midbrain (VTA), a critical brain structure of the motivational-reinforcement circuit (Wise and Bozarth, 1987), thus allowing us to evaluate common features and differences in neural response to NIC at different brain levels.

\section{MATERIALS AND METHODS}

\section{Animals and Housing}

A total of 15 male Long-Evans rats $(440 \pm 20 \mathrm{~g})$ supplied by Charles River Laboratories (Greensboro, NC) were used. Rats were housed individually under standard laboratory conditions (12-h light cycle beginning at 07:00 hours) with free access to food and water. Protocols were performed in compliance with the Guide for the Care and Use of Laboratory Animals (NIH, Publication 865-23) and were approved by the NIDA-IRP Animal Care and Use Committee.

\section{Surgery and Post-Operative Recovery}

Each rat was surgically prepared for chronic EEG and EMG as described previously in detail (Kiyatkin and Smirnov,
2010). Under general anesthesia (equithesin $0.33 \mathrm{ml} / 100 \mathrm{~g}$ i.p.; dose of sodium pentobarbital $32.5 \mathrm{mg} / \mathrm{kg}$ and chloral hydrate $145 \mathrm{mg} / \mathrm{kg}$ ), rats were implanted with three stainless steel screws threaded into the skull (two active screws on the left side: A-L, 4.0-2.0 $\mathrm{mm}$ and $\mathrm{P}-\mathrm{L}, 4.0-1.5 \mathrm{~mm}$, and a ground screw on the right side: A-L, 2.0-2.0 mm, according to Paxinos and Watson, 1998) and two stainless steel EMG electrodes implanted bilaterally in deep neck muscle. In addition, an insulated stainless steel electrode (with $0.5 \mathrm{~mm}$ open active area at the tip) was implanted in the VTA (P-L, 5.5-2.0 mm, depth $8.4 \mathrm{~mm}$ with $10^{\circ}$ angle). Screws with extension wires and gold-pin connectors were purchased from Pinnacle Technologies (Lawrence, KS) and EMG electrodes were custom made from four insulated $50 \mu \mathrm{m}$ wires, which were twisted together and covered (except the last $0.5 \mathrm{~mm}$ at the top) by a plastic catheter. This design provides strong and flexible electrodes that allow stable EMG recording for the duration of the experiment. After implantation, all six connectors were inserted into a plastic socket and fixed with dental acrylic as a head mount. During the same surgical session, each rat was implanted with a chronic jugular catheter, which ran subcutaneously to the head mount and was secured with dental acrylic in the head mount. After a 3-4-day period of recovery and habituation to the experimental chamber, recording sessions were held once daily over the next 5-8 days.

\section{EEG/EMG Recording}

Electrical signals were recorded differentially. For cortical recordings, we used two active screws threaded into the skull on the left side and a ground screw implanted on the right side. For VTA recordings, we used an electrode implanted in the VTA and the most frontal screw on the left side (A-L, $4.0-2.0 \mathrm{~mm}$ ). The ground screw was also used as a reference electrode for differential EMG recording, with two active EMG electrodes implanted bilaterally in deep neck muscle. Electrical activity from EEG and EMG electrodes passed through a pre-amplifier (Pinnacle Technologies) incorporated inside of an extension cord and electrical swivel to the main amplifiers (P15-D for cortical EEG, P55 for VTA, EEG, and EMG; Grass Electronics, West Warwick, RI), which were used for additional signal amplification and filtering. EEG and EMG signals were band pass filtered from 1 to $100 \mathrm{~Hz}$ and 100 to $1000 \mathrm{~Hz}$, respectively. The filtered signals were then passed to a Micro 1401 MK2 interface (Cambridge Electronic Design, Cambridge, UK), allowing its acquisition, recording, and analysis using a Spike2 interface (Cambridge Electronic Design).

\section{Experimental Protocol}

Experiments were conducted in an electrically insulated cage $\left(38 \times 47 \times 47 \mathrm{~cm}^{3}\right)$ placed inside a sound- and lightattenuated box $\left(60 \times 56 \times 70 \mathrm{~cm}^{3}\right)$ with electromagnetic insulation. Recordings were conducted under continuous weak white light illumination $(20 \mathrm{~W})$ in view of a small USB camera mounted above the cage. After placement in the cage, the socket on the rat's head was connected to the recording cable and a plastic catheter extension. This catheter extension was connected to a liquid swivel and an 
additional catheter extension, allowing stress-free drug delivery from outside of the cage and minimizing possible detection of the i.v. injection procedure by the animal. Each rat was intensively habituated to the recording environment both before (2-3 daily sessions of 5-7 h each) and after (2-3 sessions) surgery. The first recording session served as an additional habituation session, during which the rat was observed and only saline was injected.

Each rat was exposed to several i.v. saline injections $(0.15 \mathrm{ml}$ over $15 \mathrm{~s})$ during the next two recording sessions. Afterwards, we examined the effects of either i.v. NIC ([-]nicotine hydrogen tartrate dissolved in saline, Sigma, St. Louis, MO) or i.v. NIC-PM (NIC-PM dissolved in saline; synthesized by Dr Yi Zhang (National Institute of Mental Health, NIH) and kindly provided to us by Dr B Vaupel) at low doses $(10 \mu \mathrm{g} / \mathrm{kg}, 0.15 \mathrm{ml}$ over $15 \mathrm{~s})$ over the next 1-2 recording sessions. All doses were calculated as drug bases. During the next recording session, the rats received 3-4 saline injections. After each drug session, catheters were flushed with saline to exclude the possibility of contamination. During the next 1-2 sessions, rats received the same drugs at higher dose $(30 \mu \mathrm{g} / \mathrm{kg}$, i.v., $0.15 \mathrm{ml}$ over $15 \mathrm{~s})$. This dose range $(10-30 \mu \mathrm{g} / \mathrm{kg} /$ injection) is optimal for maintaining i.v. NIC self-administration in rats (Cox et al, 1984; Donny et al, 1995) and is comparable with that delivered during smoking of one to two cigarettes (Berridge et al, 2010; Rose et al, 1999, 2010). The intervals between drug injections were at least $90 \mathrm{~min}$ for the low dose and $120 \mathrm{~min}$ for the higher dose. The effects of NIC and NIC-PM were evaluated in different rats $(n=6$ and 7 , respectively).

In four rats that did not receive previous NIC treatment, we examined changes in EEG and EMG induced by i.v. NIC $(30 \mu \mathrm{g} / \mathrm{kg})$ following blockade of peripheral nicotinic receptors induced by hexamethonium bromide (HEXA, Sigma). After one control test with NIC $(30 \mu \mathrm{g} / \mathrm{kg})$ in drugfree conditions, rats received an i.v. injection of HEXA $(5 \mathrm{mg} / \mathrm{kg}$ dissolved in saline, $0.15 \mathrm{ml}$ over $25 \mathrm{~s})$ that followed by an NIC injection made within 10-15 min. These short post-HEXA time intervals were used because of a relatively short duration of action of this drug (Byck, 1961; Toyama et al, 1975). Similar tests (HEXA-NIC) were repeated up to three times within the session with at least 120 min intervals following each NIC injection. To minimize drug contamination, two separate catheter extensions were used in these experiments.

In six rats, which received previous NIC treatment, we also examined how EEG and EMG changes induced by NIC are affected by general anesthesia. Anesthesia was induced by urethane $(1.25 \mathrm{~g} / \mathrm{kg}$, i.p.), and the effects of i.v. NIC $(30 \mu \mathrm{g} / \mathrm{kg})$ were tested before and three times during anesthesia $(+60,150,240 \mathrm{~min})$. The data obtained during anesthesia were combined in one group and compared with control data obtained in non-anesthetized conditions. The session with urethane was always the last recording session and, after its completion, rats were euthanized.

All drug injections were made when the rat was in quiet wakefulness or drowsiness/sleep state (see Results) with no overt movements during the $120 \mathrm{~s}$ preceding each drug or saline injection. Cases in which we observed movements or sudden changes in EEG or EMG during the pre-stimulus period were removed from analysis.

\section{Data Analysis}

For each drug/saline test, EEG and EMG signals were analyzed with 5-s time resolution during 12-min recording durations, with $2 \mathrm{~min}$ before and $10 \mathrm{~min}$ after each drug/saline injection. The 5-s temporal resolution of data analysis is optimal for detecting rapid changes in electrophysiological parameters, and the 10-min post-injection interval covers the time when NIC has its primary physiological effects. For each test, we determined changes in EEG and EMG total powers (filtered within $2-58$ and $58-1000 \mathrm{~Hz}$, respectively) and changes in power of individual EEG frequencies $(\delta(2-4 \mathrm{~Hz}), \theta(4-8 \mathrm{~Hz}), \alpha$ $(8-15 \mathrm{~Hz}), \beta(15-29 \mathrm{~Hz})$, and $\gamma(29-58 \mathrm{~Hz}))$. Since EEG and EMG signals in individual rats differ in their magnitude, absolute values of total power were transformed into relative changes, taking a basal value (mean for $60 \mathrm{~s}$ pre-injection) as $100 \%$. Changes in each individual wave power were analyzed in percents with respect to the pre-event baseline determined for $60 \mathrm{~s}$ pre-injection. While EEG total power is an integral index of electrical activity, dependent upon both signal amplitude and frequency, the powers of individual EEG waves reflect their respective proportions in the EEG total power calculated for specified signal frequencies; the sum of all five EEG wave powers equals EEG total power (100\%). Since EMG signals showed robust and highly variable increases following drug administrations, EMG total power was analyzed statistically as natural logarithmic derivatives.

One-way analysis of variance (ANOVA) with repeated measures (followed by Fisher post hoc test) was used as a primary tool for evaluating statistical significance of EEG and EMG changes. Student's $t$-test was used for betweengroup comparisons. The use of the words 'increase,' 'decrease,' and 'significant' refers to statistically significant change in the parameter or differences between the compared groups or conditions (with at least $p<0.05$ ) revealed by either ANOVA or Student's $t$-test.

\section{RESULTS}

\section{Data Sample}

Data were obtained in 15 freely moving rats habituated to the recording cage and tested during several (4-6) recording sessions (a total $n=48$ ); 134 individual recordings were analyzed. After the initial period of behavioral activation following transfer from the animal facility to the recording cage $(1-2 \mathrm{~h})$, rats became quiet, showing periods of full inactivity intermixed with periods of comfort behaviors (locomotion, grooming, rearing, etc.). These changes in spontaneous behavioral activity were consistently associated with fluctuations in EEG and EMG activity. During sleep-like motor inactivity, the EEG signal in both structures showed high-magnitude fluctuations (synchronization) and minimal EMG activity, but when the rat became active, the magnitude of EEG signal transiently decreased (desynchronization) and EMG activity increased. All tests were conducted during a relatively stable synchronized EEG signal and no major changes in EMG activity within 2-min baseline intervals.

\section{EEG/EMG Responses to i.v. NIC}

NIC at both doses induced rapid, powerful, and prolonged EEG desynchronization and EMG activation 
(Figures 1 and $2 \mathrm{a}-\mathrm{c}$ ). In both brain locations, EEG total power significantly decreased within the injection interval (first or second data points corresponding to $0-5$ or 5-10 s after the start of a 15-s injection) and reached nadir at 20-25 s from the injection start; the effect in the VTA was more rapid and stronger than in the cortex. The power of EEG signal began to increase slowly, but never reached basal levels within a 10-min post-injection interval. At each dose, changes in EEG total power were similar in both locations and the effects of NIC at a higher dose were more prolonged and less variable than those at a lower dose. NIC at both doses induced strong EMG activation, which was clearly larger at a higher dose. These changes were also very rapid, with significant increases at the first or second data points (0-5 or $5-10 \mathrm{~s})$. With a $30-\mu \mathrm{g} / \mathrm{kg}$ dose, the increase in EMG total power was slower than the EEG decrease, peaking at 30-35 s post-injection. Although saline administration also induced rapid EEG desynchronization and EMG activation, these effects were much lower in both the magnitude and duration than those induced by NIC in each dose (Figures 1 and 2; Table 1).

NIC-induced changes in EEG total power were associated with robust and differential changes in individual EEG frequencies (Figure $2 \mathrm{~d}-\mathrm{h}$ ). In both structures, high-frequency $\beta$ and $\gamma$ activities strongly increased (Figure $2 \mathrm{~g}$ and $\mathrm{h}$ ) and $\alpha$ activity decreased (Figure $2 \mathrm{f}$ ). The effects were clearly dose dependent, showing stronger changes at a larger dose. These changes also had short onset latencies, reaching significance at the second (5-10s) or third (10-15s) data points following the injection onset. While the increases in $\beta$ and $\gamma$ powers were prolonged, with no return to baselines at the end of analysis interval (10 min), decrease in $\alpha$ power was more transient. These three changes were common to both structures, but they were clearly stronger in the VTA than in cortex. However, a strong tonic decrease in $\delta$ activity seen in the cortex (d1) was much less evident in the VTA (d2), not reaching the level of statistical significance. Finally, $\theta$ power significantly and dose dependently decreased in the VTA, but no change or even a slight phasic increase (significant only for $30 \mu \mathrm{g} / \mathrm{kg}$ dose) in this wave was seen in the cortex (e1 and e2).

Saline injection also resulted in rapid redistribution of EEG signal frequencies in both brain structures (solid line in Figure $2 \mathrm{~d}-\mathrm{h}$ ). Similar to that seen with NIC, $\alpha$ activity phasically decreased, but $\beta$ and $\gamma$ activities phasically increased. While these changes were equally rapid to those seen with NIC, reaching significance during the injection duration, they were incomparably weaker and shorter in

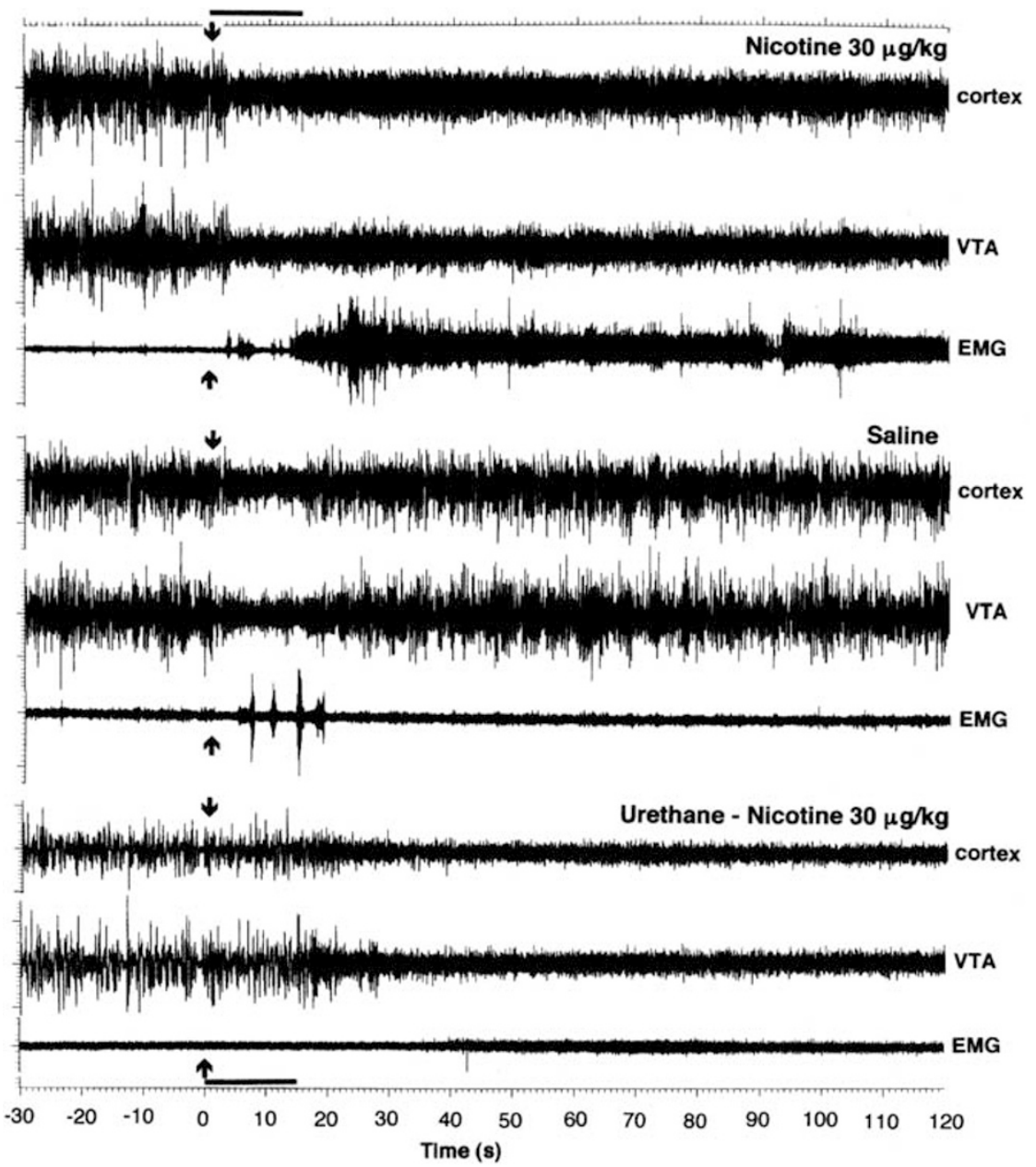

Figure I Original examples of changes in electrical activity in the cortex, VTA, and neck muscle (EMG) following i.v. injections of NIC and saline. The bottom graphs show response to i.v. NIC during urethane anesthesia. The data are shown for 30 s preceding and 120 s following each injection. Arrows at $0 \mathrm{~s}$ show the injection onset, and the duration of injection ( $15 \mathrm{~s}$ ) is shown as bold horizontal line at time axis. 

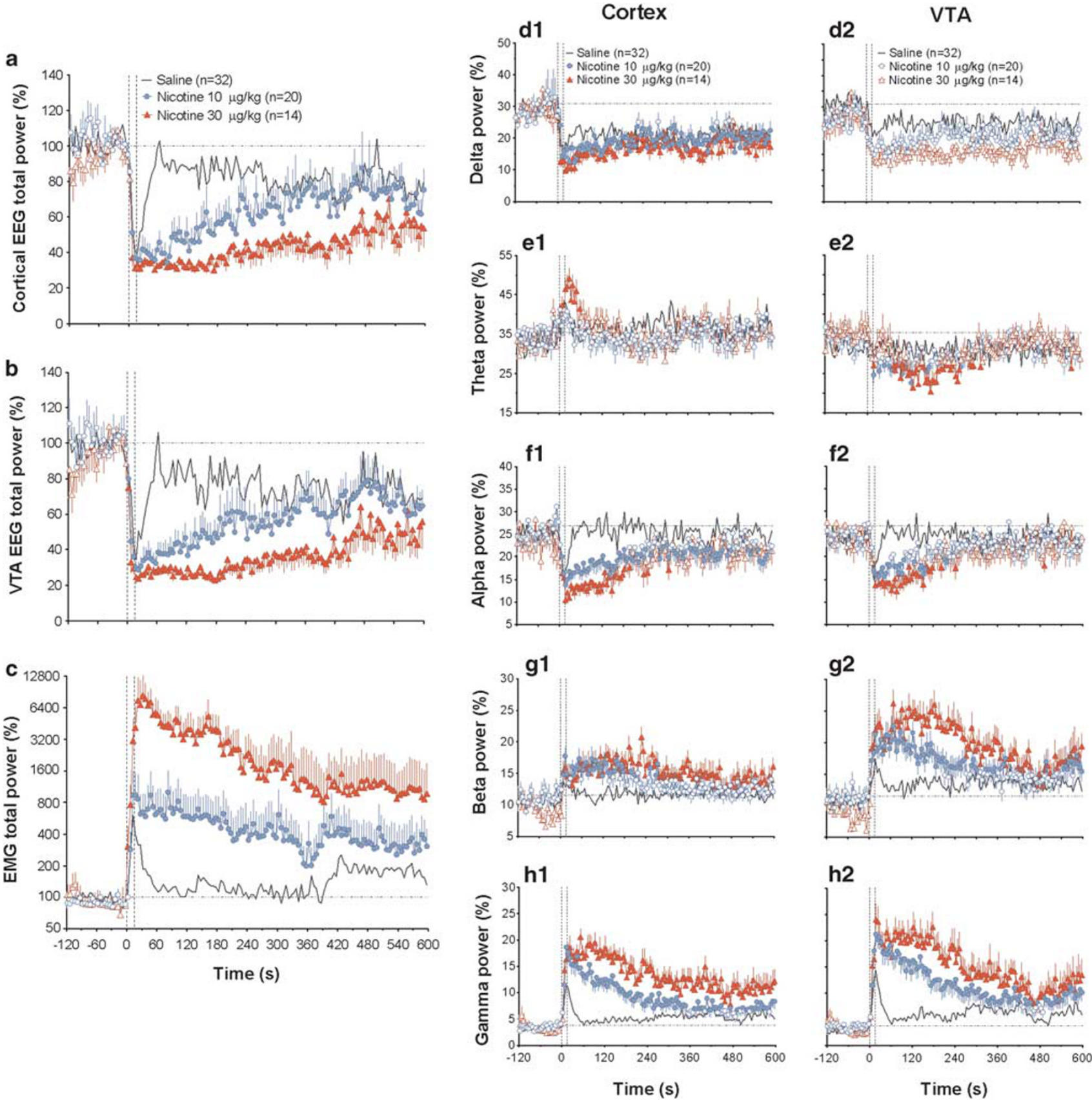

Figure 2 Mean $( \pm$ SEM) changes in EEG total powers (a, b), EMG total power (c), and individual EEG waves (d-h) induced by i.v. NIC $(I 0$ and $30 \mu g / k g$, shown as blue and red curves, respectively) and saline (gray curve) in freely moving rats. EEG and EMG total powers are expressed in \% and In(\%), respectively, with $100 \%$ = basal value determined for 60-s pre-injection (hatched lines). Changes in each individual wave power are expressed in percents from EEG total power (=100\%); baselines were determined for $60 \mathrm{~s}$ pre-injection and shown as horizontal hatched lines. Vertical hatched lines delineate the onset and offset of I5-s injection. $n=$ number of averaged tests. A one-way ANOVA with repeated measures revealed a significant effect of time (drug) for each parameter (for NIC $10 \mu \mathrm{g} / \mathrm{kg}$ : cortex total power $\mathrm{F}_{19,2280}=4.19$, VTA total power $=3.6 \mathrm{I}$, EMG total power $=1.95$; for $\mathrm{NIC} 30 \mu \mathrm{g} / \mathrm{kg}$ : $F_{13,1560}=3.94,3.87$, and 5.45; for saline: $F_{31.3720}=2.45,1.82$, and 1.90; each $\left.p<0.05\right)$. For $\mathrm{NIC} 10 \mu g / \mathrm{kg}$, the effects in the cortex were significant with respect to $\delta\left(\mathrm{F}_{19,2280}=1.60\right), \alpha(=1.84), \beta(=2.43)$, and $\gamma(=7.96)$ activities, but absent for $\theta$ activity $(=1.01$, no significance). For the same dose in the VTA, the effects were significant with respect to $\theta\left(\mathrm{F}_{19.2280}=1.46\right), \alpha(=2.09), \beta(=2.63)$, and $\gamma(=6.96)$ activities, but absent for $\delta$ activity $(=1.15$, no significance). For NIC $30 \mu \mathrm{g} / \mathrm{kg}$, the effects in the cortex were significant with respect to all waves $\left(\delta: \mathrm{F}_{13,1560}=2.03, \theta=2.42, \alpha=3.1 \mathrm{I}, \beta=1.7 \mathrm{I}\right.$, and $\gamma$ $=4.69)$. For NIC $30 \mu \mathrm{g} / \mathrm{kg}$, the effects in the VTA were also significant with respect to $\theta\left(F_{13,1560}=2.04\right), \alpha(=2.7 \mathrm{I}), \beta(=4.04)$, and $\gamma(=5.35)$ activities, but absent for $\delta$ activity $(=1.19$, no significance). While the effects of NIC were evaluated for the entire duration of post-injection interval ( 10 min), a shorter interval (I20s) was used for evaluating the effects of saline. Since saline effects are strong but transient, analysis over a long period of time underestimates the phasic effects of saline. The effects of saline were significant for all cortical EEG waves $\left(\delta: \mathrm{F}_{31,744}=2.76, \theta=2.67, \alpha=4.94, \beta=2.19\right.$, and $\gamma=12.84)$ and all VTA waves $\left(\delta: \mathrm{F}_{13,744}=1.8 \mathrm{I}, \alpha=3.63, \beta=2.90\right.$ and $\left.\gamma=10.86\right)$, except $\theta$ wave $(=1.18$, no significance). Filled symbols indicate values significantly different from baseline by Fisher's LSD post hoc test. 
Table I Mean Changes in Rapid and Long-Term Components of EEG and EMG Responses Induced by Drugs Used in This Study

\begin{tabular}{|c|c|c|}
\hline Parameters & $\begin{array}{l}\text { Rapid changes } \\
\quad(5-30 \mathrm{~s})\end{array}$ & $\begin{array}{l}\text { Long-term changes } \\
\quad(30-300 \mathrm{~s})\end{array}$ \\
\hline \multicolumn{3}{|l|}{ EEG, \% } \\
\hline \multicolumn{3}{|l|}{ Cortex } \\
\hline Saline & $50.59 \pm 5.00$ & $86.19 \pm 3.17$ \\
\hline $\mathrm{NIC} 10$ & $38.48 \pm 2.30 *$ & 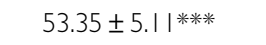 \\
\hline NIC 30 & $33.09 \pm 1.68 * * * *$ & $36.91 \pm 2.78^{* * * * *}$ \\
\hline NIC 30+urethane & $72.23 \pm 3.29 * *, \dagger \dagger$ & $53.1| \pm 6.1|$ ****** \\
\hline NIC 30+HEXA & $41.89 \pm 3.21^{\dagger}$ & $62.92 \pm 6.65^{* * *},+\dagger \dagger$ \\
\hline NIC-PM 10 & $32.69 \pm 2.03 * *$ & $73.53 \pm 5.04 *+\dagger \dagger$ \\
\hline NIC-PM 30 & $44.4 \mathrm{I} \pm 3.25^{\dagger \dagger}$ & $72.25 \pm 4.10^{*},+\dagger \dagger$ \\
\hline \multicolumn{3}{|l|}{ VTA } \\
\hline Saline & $45.65 \pm 3.59$ & $78.54 \pm 2.99$ \\
\hline $\mathrm{NIC} 10$ & $31.85 \pm 1.37$ *** & $47.35 \pm 4.98$ ***** \\
\hline NIC 30 & $26.22 \pm 1.64 * * * * *$ & $29.27 \pm 2.58$ ***** \\
\hline NIC 30+urethane & $77.47 \pm 7.85 * *, \dagger \dagger$ & $42.99 \pm 6.50$ ***** \\
\hline NIC 30+HEXA & $43.71 \pm 4.96^{\dagger \dagger \dagger}$ & $61.22 \pm 7.46 * t+\dagger$ \\
\hline NIC-PM IO & $27.33 \pm 1.34 * *$ & $67.44 \pm 6.77^{\dagger \dagger \dagger}$ \\
\hline NIC-PM 30 & $35.53 \pm 1.83 *$ & $64.96 \pm 5.39^{\dagger \dagger \dagger}$ \\
\hline \multicolumn{3}{|l|}{ EMG, In(\%) } \\
\hline Saline & $5.83 \pm 0.19$ & $4.81 \pm 0.18$ \\
\hline $\mathrm{NIC} 10$ & $6.67 \pm 0.27$ 米米 & $6.28 \pm 0.43 * *$ \\
\hline $\mathrm{NIC} 30$ & 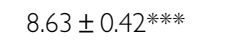 & $8.07 \pm 0.29 * * * *$ \\
\hline NIC 30+urethane & $4.66 \pm 0.14 * * *,+\dagger \dagger$ & $4.73 \pm 0.09^{\dagger \dagger \dagger}$ \\
\hline NIC 30+HEXA & $7.29 \pm 0.38 * * * *, \dagger$ & $6.18 \pm 0.41$ **⿻, \\
\hline NIC-PM IO & $6.38 \pm 0.17^{*},+\dagger \dagger$ & $5.15 \pm 0.43^{\dagger \dagger \dagger}$ \\
\hline NIC-PM 30 & $6.95 \pm 0.19 * * *,+\dagger \dagger$ & $5.44 \pm 0.54^{\dagger \dagger \dagger}$ \\
\hline
\end{tabular}

Each value represents a mean value of total power ( \pm SEM) calculated for distinguished time intervals. Asterisks define statistical significance of differences vs saline $\left(* p<0.05\right.$, *** $p<0.01$, and $\left.{ }^{* * *} p<<0.001\right)$ and ${ }^{\dagger}$ define statistical differences vs NIC $30 \mu \mathrm{g} / \mathrm{kg}\left({ }^{\dagger} p<0.05 ;{ }^{\dagger \dagger} p<0.01\right.$, and ${ }^{\dagger \dagger} p<0.00$; ; Student's $t$-test). The numbers of tests in each group are shown in figures.

their durations. Similar to that seen with NIC, in the cortex $\delta$ activity rapidly decreased and $\theta$ activity phasically increased. While decrease in $\delta$ activity was also seen in the VTA, saline injection did not affect $\theta$ activity in this structure. Similar to NIC, changes in high-frequency $\beta$ and $\gamma$ activities in the VTA were stronger than those in the cortex.

Since the injection of pharmacologically neutral saline induces rapid, transient EEG desynchronization, and EMG activation, effect of NIC on these parameters includes a non-pharmacological component. Thus, to evaluate the relative contribution of pharmacological $v s$ non-pharmacological factors in mediating the EEG and EMG effects of i.v. NIC, we analyzed the time course of their difference (ie, NIC-saline) both for integral measures of EEG and EMG responses and individual EEG waves (Figure 3).

Between-group differences in cortical and VTA EEG total powers (Figure $3 \mathrm{a}$ and $\mathrm{b}$ ) become significant at 20-25 s (NIC $30 \mu \mathrm{g} / \mathrm{kg}$ ) after the start of a 15-s injection, reach maximum at $\sim 60 \mathrm{~s}$, and then dissipate slowly for the next $4-5 \mathrm{~min}$. A rapid and strong pharmacological contribution was also evident in EMG changes (Figure 3c); the difference between NIC and saline became significant even at the early time (5-10s) and maintained for the entire recording interval. Rapid appearance of pharmacological effects of NIC was even more evident in the analyses of individual EEG waves (Figure 3d-f; NIC $30 \mu \mathrm{g} / \mathrm{kg}$ ). In the cortex, the latency was minimal (5-10s) for $\gamma$ waves (Figure 3f) and slightly longer for $\beta$ and $\alpha$ waves (10-15 and 15-20s, respectively) (Figure $3 \mathrm{~d}$ and $\mathrm{f}$ ). The effects of drug were maximal at $\sim 1-2 \mathrm{~min}$ after the injection and the difference $v s$ saline maintained for 5-6 min post-injection. Similarly, rapid differences were found in the VTA $(5-10,10-15$, and $10-15 \mathrm{~s}$ for $\gamma, \beta$, and $\alpha$ frequencies).

\section{EEG and EMG Responses to i.v. NIC During Urethane Anesthesia: Test for Sensory Mechanism}

To test for involvement of sensory mechanisms in the effects of i.v. NIC, we examined how NIC-induced EEG and EMG responses are changed during urethane anesthesia. Differences in electrophysiological effects of i.v. NIC $(30 \mu \mathrm{g} / \mathrm{kg})$ in two conditions (anesthesia and no anesthesia) were assessed in EEG and EMG total powers (Figure 4a-c), mean values of rapid and long-term components of EEG and EMG responses (Table 1), and individual EEG waves (Supplementary Figure S1).

The i.v. NIC injected during urethane anesthesia had significant and strong effects of cortical and VTA EEG total powers, but the motor effect was fully blocked (Figure 4). However, in contrast to unanesthetized conditions, EEG desynchronization in both structures developed with longer onset latencies, was much more gradual, but eventually reached the same strength 2-3 min after the NIC injection (see original example in Figure 1). The rapid EEG change was not only significantly weaker than that with NIC, but it was also significantly weaker than that of saline tested in control conditions (Table 1). In contrast, long-term effects of NIC on EEG were equal in both conditions. A similarly delayed response to NIC was also found in analysis of individual EEG waves, which had a different wave distribution in baseline compared with unanesthetized conditions (see Supplementary Figure S1). During anesthesia, in both the cortex and VTA, $\delta$ activity (a1 and a2) was much more prominent $(p<0.001)$ and $\alpha$ activity (c1 and c2) less pronounced than in control $(p<0.001)$ (Supplementary Figure S1a and $c$ ). In both structures, the EEG signal during anesthesia did not differed from control in $\beta$ power ( $\mathrm{d} 1$ and $\mathrm{d} 2$ ), but had a higher proportion of $\gamma$ power (e1 and e2; $p<0.05)$.

Despite differences in basal $\delta$ power, this EEG frequency also strongly decreased following i.v. NIC injection. However, this decrease developed more slowly, but eventually became as strong as in control conditions (Supplementary Figure S1a). In contrast, $\alpha$ activity did not phasically change following NIC injection during anesthesia (c). Despite longer latencies and slower peaking, highfrequency $\beta$ and $\gamma$ activities strongly increased following NIC injection ( $d$ and e). Finally, NIC during anesthesia strongly decreased $\theta$ activity in both structures (b), but differential responses (phasic increases in cortex and decrease in VTA) were seen in control.

To examine which components of NIC-induced EEG response are affected by general anesthesia, we analyzed the time course of differences between changes in cortical EEG 

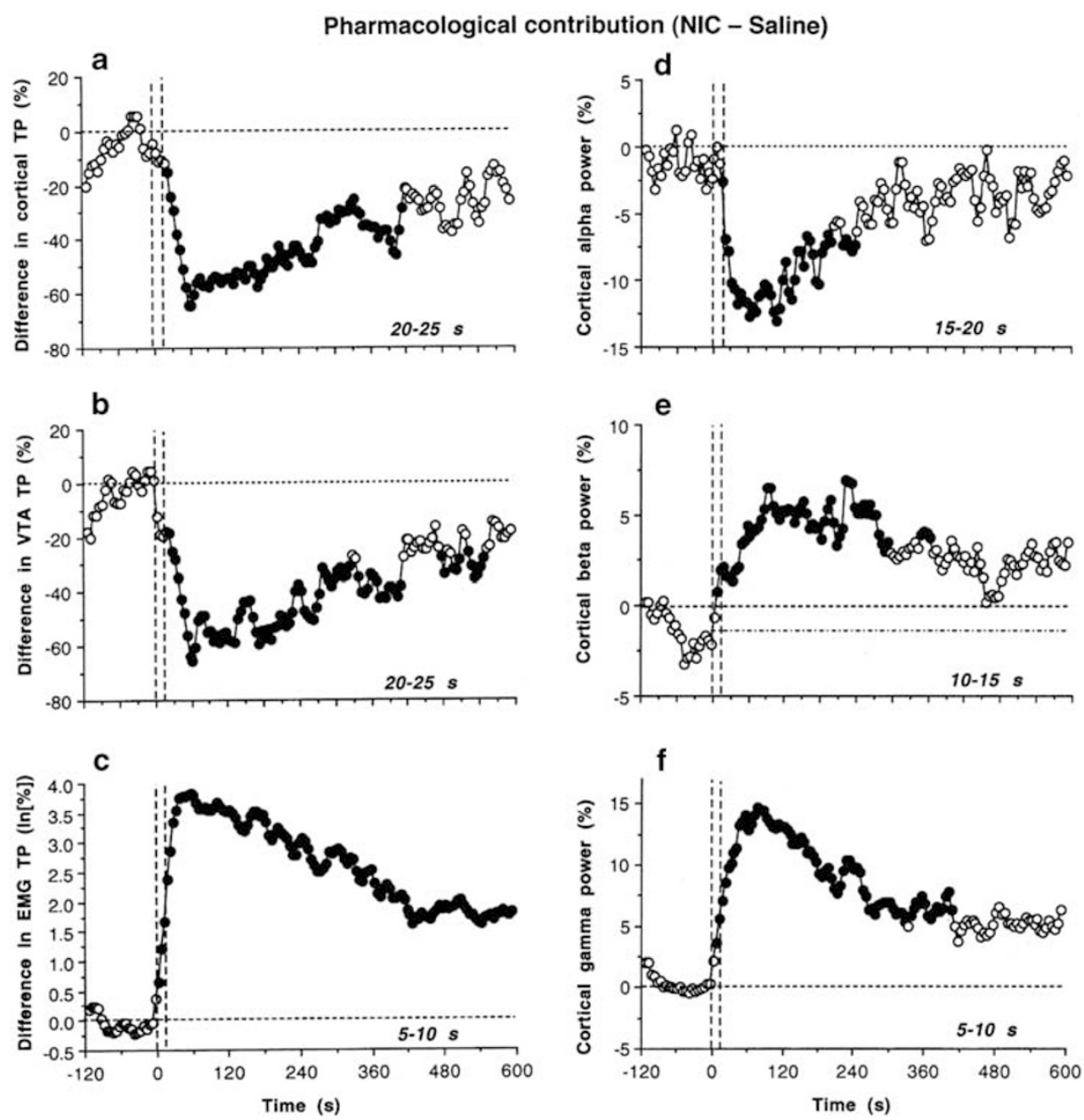

Figure 3 Pharmacological contributions to NIC-induced EEG desynchronization and EMG activation. Each graph represents the difference in parameters (a, EEG total power; b, VTA total power; c, EMG total power; d, cortical $\alpha$ power; e, cortical $\beta$ power; f, cortical $\gamma$ power) between NIC $30 \mu g / k g$ and saline. Values with significant between-group differences $(p<0.05$; Student's $t$-test) are shown as black filled symbols. Time intervals corresponding to the first significant differences (ie, prevalence of pharmacological over non-pharmacological factors) are specified in right bottom corner of each graph. Vertical hatched lines at 0 and $15 \mathrm{~s}$ indicate the duration of injection, horizontal hatched lines at $0 \%$ represent a zero difference between groups.

total power induced by i.v. NIC $(30 \mu \mathrm{g} / \mathrm{kg})$ in urethane and control conditions. As clearly seen in Figure 5a, general anesthesia drastically reduces the rapid components of NICinduced EEG desynchronization, without affecting later components. The between-group difference appears immediately after the injection onset, becomes significant from 5 to $10 \mathrm{~s}$, peaks at $\sim 10-20 \mathrm{~s}$, and disappears at $\sim 75 \mathrm{~s}$. Similar changes were also seen in the VTA (data not shown). The blockade of the initial component of EEG desynchronization is also clearly evident in comparing differences in the effects of NIC during anesthesia and saline (b). This difference (ie, the component that is blocked by general anesthesia) is evident at 5-20 s after the injection start, but is rapidly inverted, showing the appearance (from $\sim 40$ s) of pharmacological effect of NIC.

\section{EEG and EMG Responses to i.v. NIC During Blockade of Peripheral Nicotinic Receptors by HEXA}

To evaluate the contribution of peripheral actions of NIC in mediating its effects on EEG and EMG, we used HEXA, a drug that selectively blocks the peripheral pool of nACh receptors without affecting their central pool. While i.v. HEXA injection alone induced weak, transient motor activation and EEG desynchronization, the activity quickly ceased and rats at 8-12 min post-injection were hypoactive, typically showing a high-magnitude, sleep-like EEG activity in both structures. Thus, we tested the effects of i.v. NIC after HEXA pre-treatment within this time scale.

The effects of NIC following HEXA pre-treatment were reduced as a whole, with a minimal (but significant) effect on rapid components and a powerful, highly significant effect on the long-term components of the EEG response (Figure 4; Table 1). Similarly, NIC-induced EMG activation was attenuated following HEXA pre-treatment, with especially strong effect on long-term changes. In contrast to prolonged motor activation induced by NIC in control conditions ( $>10 \mathrm{~min}$, see Figure 2), this response was evident only for 2-3 min after HEXA pre-treatment.

While no gross motor activity and a visual pattern of slow-wave sleep were seen at 10-15 min after i.v. HEXA injection, the wave characteristics of this baseline state slightly differed from those in control conditions. As shown in Supplementary Figure S2, in the HEXA group, 

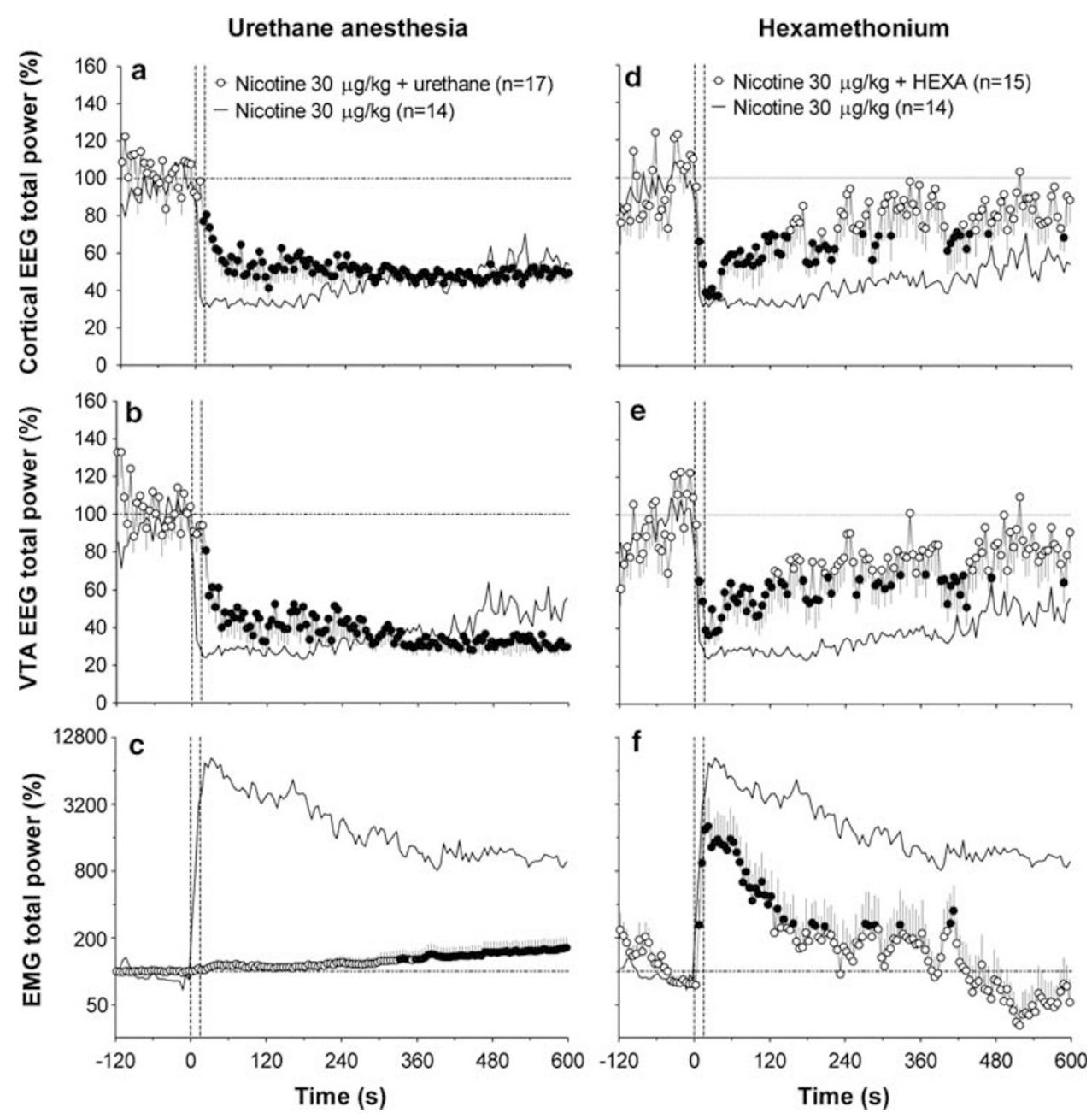

Figure 4 The effects of i.v. NIC $(30 \mu \mathrm{g} / \mathrm{kg})$ during urethane anesthesia $(\mathrm{a}-\mathrm{c})$ and blockade of peripheral nicotinic receptors by HEXA ( $\mathrm{d}-\mathrm{f})$. Graphs show mean $( \pm$ SEM) changes in cortical EEG total power $(a, d)$, VTA EEG total power (b, e), and EMG total power (c, f) induced by NIC in each experimental condition. Superimposed black lines in each graph represent the changes in parameters induced by NIC (30 $\mu / \mathrm{kg})$ in non-anesthetized (a-c) and HEXA free $(d-f)$ conditions (control). Hatched vertical lines show the onset and offset of I 5 -s injection and horizontal hatched lines display basal values. $n=$ number of averaged tests. ANOVA with repeated tests revealed a significant (at least $p<0.05$ ) effect of NIC for each parameter both during urethane anesthesia (cortex total power $\mathrm{F}_{16,1920}=3.00$, VTA total power $=4.17$, EMG total power $=2.49$ ) and after HEXA pre-treatment (cortex total power $\mathrm{F}_{14,1680}=1.86$, $\mathrm{VTA}$ total power $=1.57$, EMG total power $=7.23$ ). Filled symbols indicate values significantly different from baseline by Fisher's LSD post hoc test.

$\delta$ activity (a) was slightly but significantly reduced in both structures in baseline and did not change after NIC injection. In contrast, $\alpha$ activity (c) was more prominent after HEXA treatment and significantly decreased by NIC. However, this decrease was more transient than in controls. Basal $\beta$ and $\gamma$ activities (d and e) after HEXA were slightly more prominent in the cortex and similar to control in the VTA. Although NIC after HEXA pre-treatment increased $\beta$ and $\gamma$ frequencies ( $d$ and $e$ ), both the magnitude and duration of these responses were strongly reduced in both structures. In contrast to powerful $\beta$ activation in control, this effect was virtually fully absent after HEXA (d). This attenuating effect of HEXA was especially strong in the VTA, which showed larger changes in highfrequency waves than the cortex under control conditions (compare $\mathrm{d}$ and e). Finally, $\theta$ activity was similar in both conditions and no NIC-induced changes were seen in this frequency after HEXA treatment (Supplementary Figure S2b).
To examine which components of the NIC-induced EEG response are affected by blockade of peripheral nACh receptors, we analyzed the time course of differences between changes in cortical EEG total power in HEXAtreated and control conditions (Figure 5c). Blockade of peripheral nACh receptors has an immediate but weak attenuating effect on rapid components of NIC-induced EEG desynchronization (significant difference at 5-15 s post-injection) and strong, prolonged effect on its later components (50-400 s). The difference between the effects of NIC + HEXA and saline (Figure 5d) suggests that NIC after blockade of peripheral nicotinic receptors still has an immediate effect on EEG (35-70s), but fully blocks the slow component of NIC-induced desynchronization (no difference $v s$ saline). Comparison of this graph (NIC + HEXA-saline) with that showing the same difference for regular NIC (NIC-saline, see Figure 3a) clearly demonstrates that blockade of peripheral nACh receptors drastically reduces NIC-induced desynchronization. 

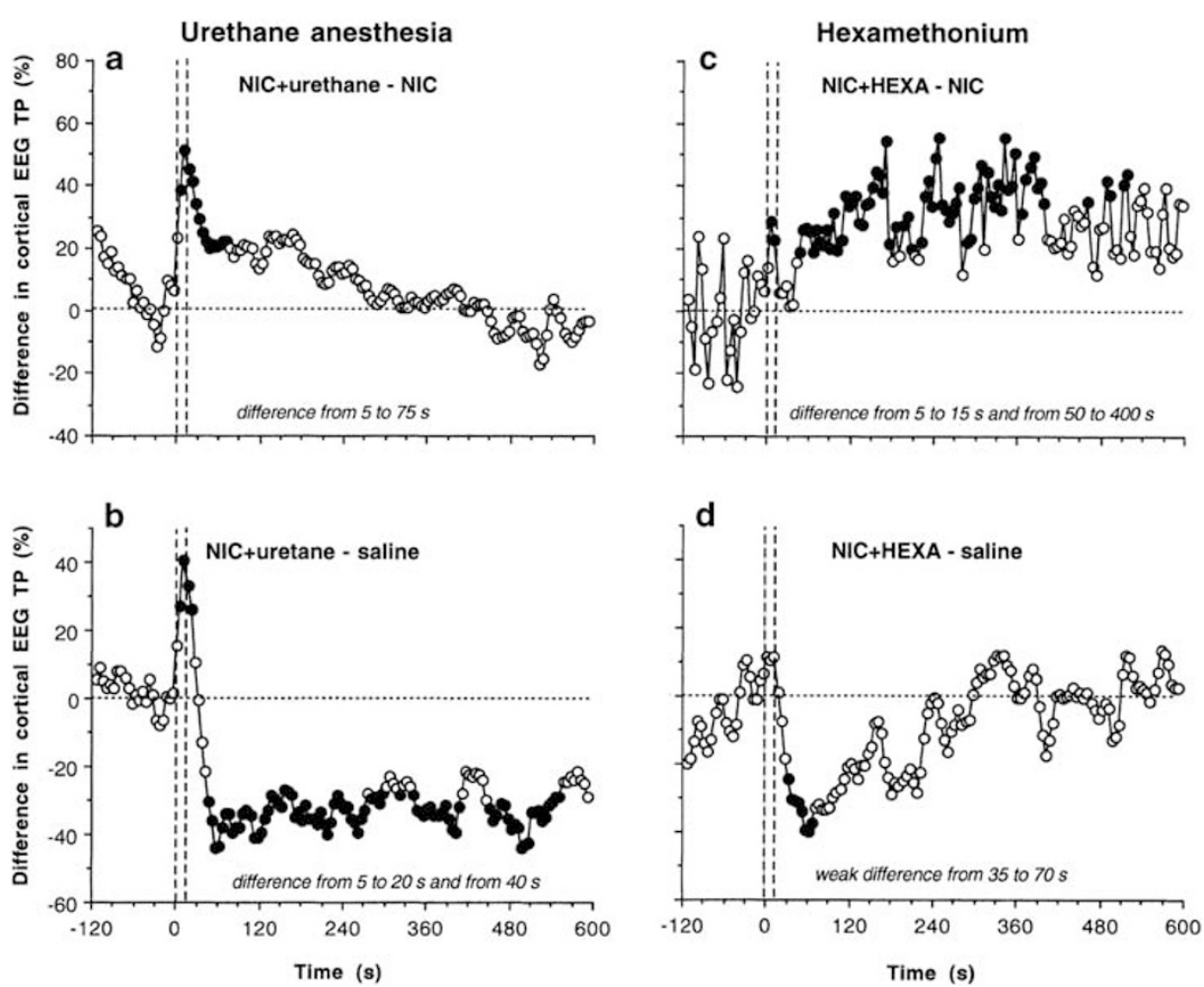

Figure 5 Contributions of sensory mechanisms (urethane anesthesia) and peripheral drug actions (HEXA) in mediating NIC-induced cortical EEG desynchronization. Left column shows differences in changes of cortical EEG total power induced by NIC (30 $\mu \mathrm{g} / \mathrm{kg})$ during anesthesia and no anesthesia (a, NIC + urethane-NIC) and NIC (30 $\mu \mathrm{g} / \mathrm{kg})$ during anesthesia and saline in unanesthetized conditions (b, NIC + urethane-saline). Right column shows differences in changes of cortical EEG total power induced by NIC $(30 \mu \mathrm{g} / \mathrm{kg})$ after HEXA $(5 \mathrm{mg} / \mathrm{kg}$, i.v.) pre-treatment and in control, drug-free conditions

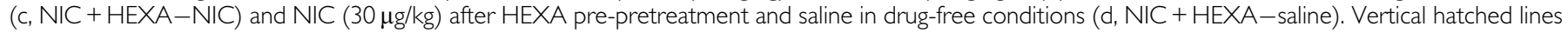
show the onset and offset of I5-s injection and horizontal hatched lines display zero between-group difference. Filled symbols indicate significant betweengroup differences ( $p<0.05$; Student's t-test). Time intervals of significant between-group differences are specified in right bottom corners of each graph. For other explanations, see the text.

\section{EEG and EMG Responses to i.v. NIC-PM}

Another approach to evaluate the contribution of peripheral actions of NIC in mediating its central effects is to use an NIC agonist that is unable to cross the BBB. In this study, we used NIC-PM, a charged BBB-impermeable NIC derivative (Aceto et al, 1983). While direct data on the affinity of this NIC analog to different subtypes of peripherally located $\mathrm{nACh}$ receptors are still lacking (see Discussion), we assumed that NIC-PM is comparable to NIC in its ability to interact with these receptors. Data from this experiment are shown in Table 1 (statistical differences) and in Figure 6.

NIC-PM at both doses tested induced rapid EEG desynchronization and EMG activation, but these effects were clearly weaker than those induced by NIC (Figure 6; Table 1). In both brain locations, EEG total power significantly decreased within the injection interval $(0-15 \mathrm{~s})$ and reached nadir at $20-25 \mathrm{~s}$ from the injection start (Figure 6a,b,d,e). Then the amplitude of the EEG signal began to increase slowly, but never reached basal levels. At each dose, changes in EEG signals were surprisingly similar in both locations. Despite the similar time course, the effects of NIC-PM were stronger at lower rather than higher doses. The effects of NIC-PM were drastically different from those of NIC and generally resembled the immediate effects of saline. However, the mean decrease in EEG total power for the rapid component of the response was significantly stronger than that of saline in both brain structures at $10 \mu \mathrm{g} / \mathrm{kg}$ dose $(p<0.01$; see Table 1$)$. This difference was weaker for $30 \mu \mathrm{g} / \mathrm{kg}$ dose, reaching significance only in the VTA. The effects of NIC-PM on EEG total power were also stronger than those of saline for long-term components of change, but this difference was significant $(p<0.05)$ only in the cortex. While mimicking the time course of EMG activation, rapid motor effects induced by NIC-PM at both doses were significantly stronger than that of saline, but much weaker than those of NIC (Figure $6 \mathrm{c}$ and f).

NIC-PM also drastically differed from NIC in changes of individual EEG waves (compare Figure 6g-i with Figure 2d-h). While in both cases, cortical $\delta$ and $\alpha$ activities decreased and $\gamma$ activity increased, the magnitude and duration of these changes were much weaker than those for NIC. In contrast to the robust and prolonged increase in $\beta$ activity induced by NIC, NIC-PM did not affect this wave at all (Figure 6h). The pattern of wave redistribution induced by NIC-PM was similar to that induced by saline (see Figure 6), but the magnitude of changes (for NIC-PM $10 \mu \mathrm{g} / \mathrm{kg}$ ) were larger, especially for $\alpha$ (Figure 6g) and $\gamma$ (Figure 6i) waves. In the last case, the difference was significant both for rapid and long-term changes. Similar changes were seen in the VTA (data not shown). 

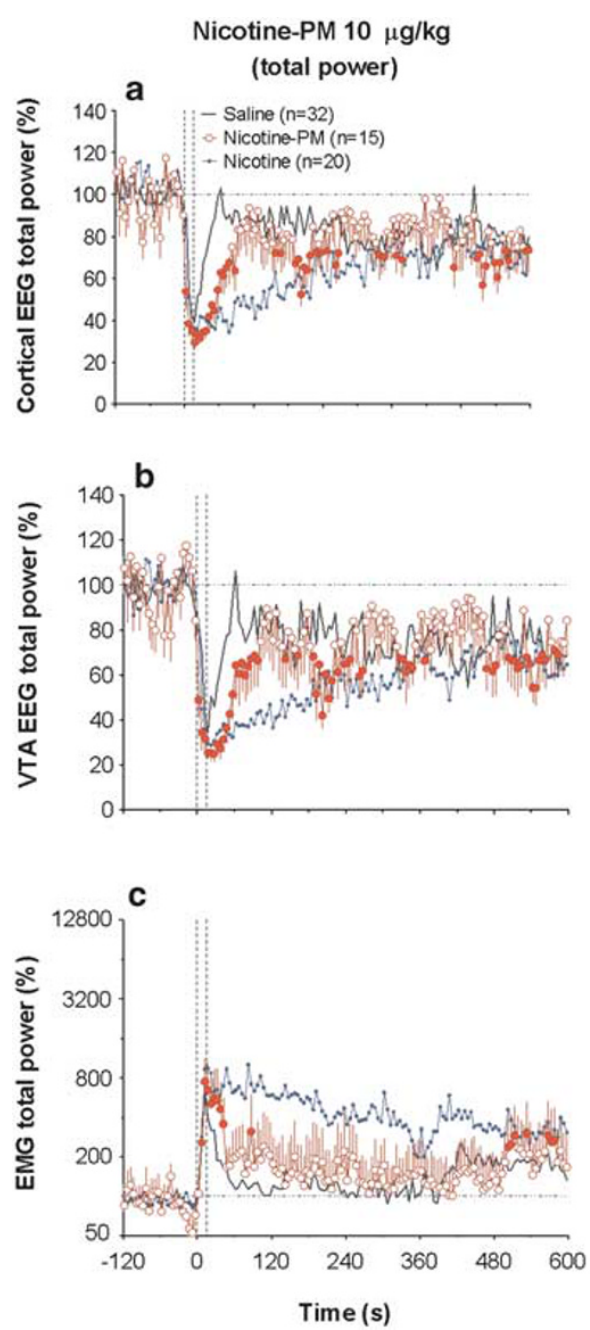
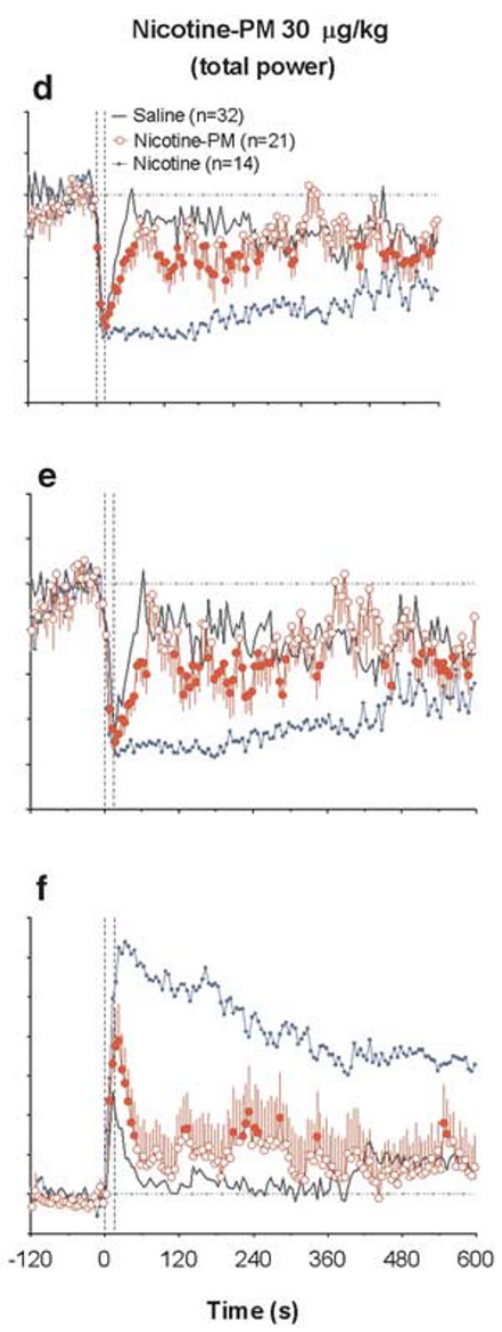
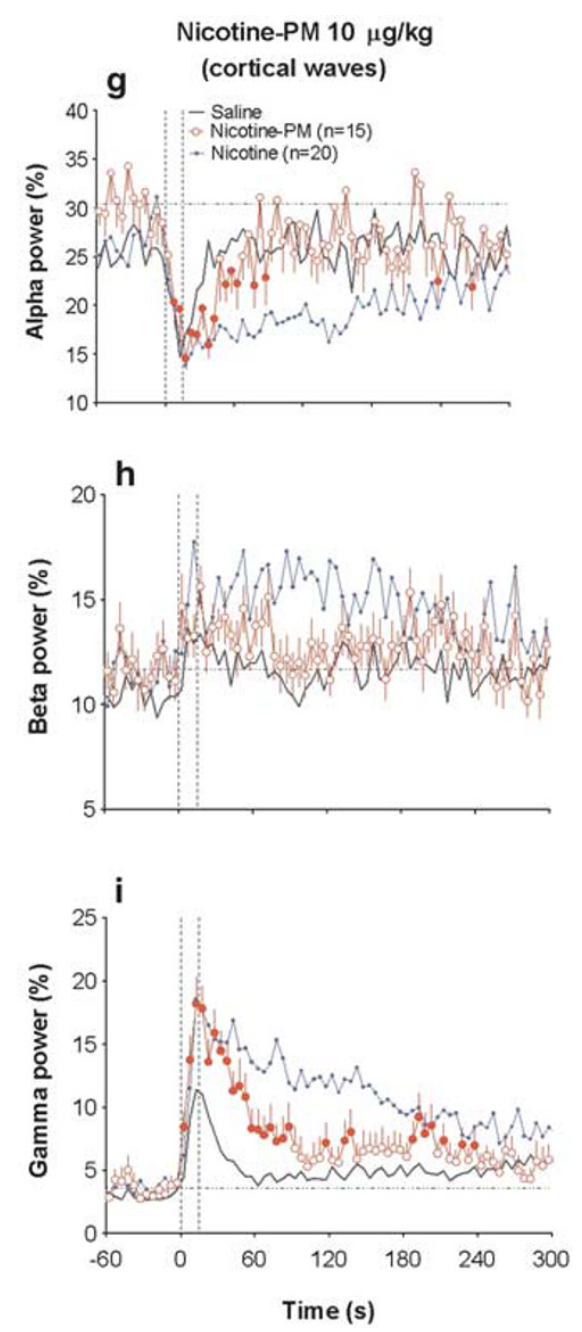

Figure 6 Mean $( \pm$ SEM) changes in EEG and EMG total powers $(\mathrm{a}-\mathrm{f})$ and selected cortical EEG waves ( $\mathrm{g}-\mathrm{i})$ induced by i.v. NIC-PM (I0 and $30 \mu \mathrm{g} / \mathrm{kg}$, red curves) in freely moving rats. EEG total power is expressed in \%, with 100\% = basal value for 60-s pre-injection. EMG total power is expressed in In(\%), with the same $100 \%$ baseline determined for 60 s pre-injection. Changes in each individual wave power are shown in percents from EEG total power ( $=100 \%$ ). Hatched vertical lines show the onset and offset of I5-s injection and hatched horizontal lines show basal values. $n=$ number of averaged tests. An ANOVA with repeated measures revealed a significant effect of time on EEG and EMG total powers (for NIC-PM at $10 \mu \mathrm{g} / \mathrm{kg}$ : cortical EEG = $\mathrm{F}_{14}$, I680 $=2.52$, $\mathrm{VTA}$ filed potentials = 2.16, EMG = I.5I; for NIC-PM $30 \mu g / \mathrm{kg}$ : $F_{20,2400}=1.93,1.77$, I.35; each $\left.p<0.00 \mathrm{I}\right)$. The effect of time for NIC-PM at I0 $\mu g / \mathrm{kg}$ was significant with respect to $\delta\left(F_{14.840}=1.48, p<0.05\right), \alpha(=2.68, p<0.01, g)$, and $\gamma(=7.38, p<0.001$, i) powers and insignificant with respect to $\theta(=0.96)$ and $\beta(=1.29)$ waves. Values significantly different from baseline are shown as filled symbols. Superimposed blue and black curves indicate changes induced by NIC and saline, respectively.

Figure 7 shows the time course of differences in the effects of NIC-PM and NIC (Figure 7a-c) as well as NIC-PM and saline (Figure $7 \mathrm{~d}-\mathrm{f}$ ). If these drugs differ only in activated nACh receptors (ie, all receptors for NIC and only peripheral receptors for NIC-PM), the difference in their effects could show the component of the response determined by selective activation of the central receptor pool. On the other hand, the difference in effects of NIC-PM and saline could show the component of the response that is determined by activation of peripheral $\mathrm{nACh}$ receptors. As shown in Figure $7 \mathrm{a}$, the between-group difference in cortical EEG (ie, prevalence of central $v s$ peripheral action) becomes significant only at $65-70 \mathrm{~s}$ and is maintained for only $\sim 70 \mathrm{~s}$ for NIC and NIC-PM used at the same lower dose $(10 \mu \mathrm{g} / \mathrm{kg})$. On the other hand, the difference between the effects of NIC-PM at $10 \mu \mathrm{g} / \mathrm{kg}$ and saline on cortical EEG (ie, prevalence of peripheral drug action $v s$ non-pharmacological actions) appeared at $15 \mathrm{~s}$ and maintained until $70 \mathrm{~s}$ after the injection start (Figure 7d). A similar pattern of changes was seen in VTA EEG (Figure 7b and e). The central contribution to the NIC's effect on VTA EEG became evident only from 100 to $105 \mathrm{~s}$ and was maintained for only $\sim 40 \mathrm{~s}$ (Figure $7 \mathrm{~b}$ ). In contrast, the peripheral contribution was dominating from 20 to $65 \mathrm{~s}$ from the injection onset (Figure 7e), the same time interval that was found in the cortex. A similar timing of central contribution was also found in EMG (Figure 7c). In this case, the difference in the effects of NIC and NIC-PM at $10 \mu \mathrm{g} / \mathrm{kg}$ appeared at $\sim 45 \mathrm{~s}$, becomes significant from 75-80 s, and was maintained for a relatively short time. Although an increase in EMG induced by NIC-PM at $10 \mu \mathrm{g} /$ $\mathrm{kg}$ was stronger than that induced by saline, the difference for each time point never reached a statistical significance (Figure 7f). 

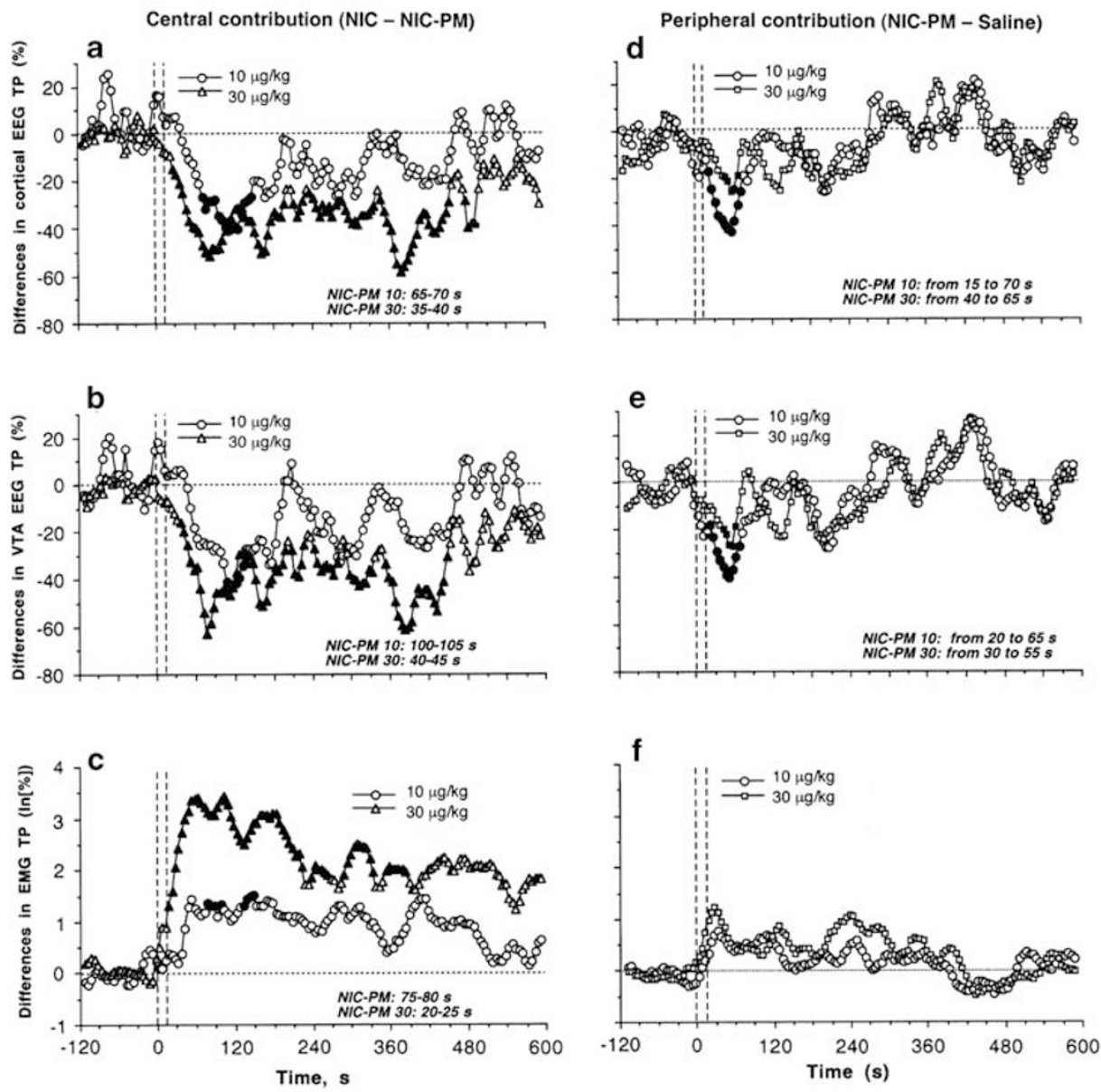

Figure 7 Central and peripheral contributions to NIC-induced EEG desynchronization and EMG activation. Left column shows differences between effects of NIC and NIC-PM (at IO and $30 \mu \mathrm{g} / \mathrm{kg}$ doses) on cortical EEG total power (a), VTA total power (b), and EMG total power (c). Right column shows differences between effects of NIC-PM (IO and $30 \mu \mathrm{g} / \mathrm{kg}$ ) and saline on cortical EEG total power (d), VTA total power (e), and EMG total power (f). Values with significant differences are shown as filled symbols. Graphs in left column show in right bottom corner the time interval of the first significant difference between NIC and NIC-PM ('central over peripheral contribution'). Graphs in right column show the time interval of significant differences between the effects of NIC-PM and saline ('peripheral drug contribution over non-pharmacological effects'). Vertical hatched lines at 0 s show the moment of injection, horizontal hatched lines at $0 \%$ show zero difference.

Interestingly, the relative contributions of central and peripheral actions of NIC were different when the effects of drugs at higher doses (tested at later sessions) were compared. In this case, central contribution becomes evident at early times (from 35, 40, and $20 \mathrm{~s}$ for cortical EEG, VTA, EEG, and EMG, respectively) and is maintained much longer (6-8 $\mathrm{min})$ than in lower doses. Similarly, the peripheral action of NICPM was evident later (30-40 s) and only for a very short time interval (20-30 s). An increase in EMG induced by NIC-PM at $30 \mu \mathrm{g} / \mathrm{kg}$ dose was slightly larger than those induced by either NIC-PM at $10 \mu \mathrm{g} / \mathrm{kg}$ or saline. However, these differences never reached statistical significance.

\section{DISCUSSION}

This study revealed that i.v. NIC at low, human-relevant doses induces rapid, strong, and relatively prolonged EEG desynchronization, which appears within the duration of a 15-s injection ( $0-5$ or 5-10 s) and peaks at about $15-25 \mathrm{~s}$ after its start. Similar to simple sensory stimuli such as sound (Kiyatkin and Smirnov, 2010), NIC in both structures induced a typical activation triad: decrease in $\alpha$ and increases in $\beta$ and $\gamma$ activities, which all reached significance level at 5-10 s after the injection start. However, in contrast to simple sensory stimuli, NIC-induced changes in EEG were much stronger and longer lasting, especially with respect to high-frequency $\beta$ and $\gamma$ activities. These robust changes in EEG were tightly related to increases in EMG activity, which became significant at the same time scale (5-10s), peaked rapidly, and returned slowly toward baseline. Interestingly, cortical EEG and VTA field potential activity were quite similar in both basal wave distribution and in the timing and frequency characteristics of the NICinduced response, suggesting a generalized pattern of neural activation. However, changes in VTA field potentials were consistently stronger and more prolonged than in the cortex and they had specific changes in $\delta$ and $\theta$ frequencies. Although it is known that NIC easily crosses the BBB and could reach the brain within 1 min following i.v. administration (Berridge et al, 2010; Rose et al, 2010), such a rapid dynamics of electrophysiological effects could suggest a peripheral action of NIC as a possible trigger of generalized EEG activation and subsequent EMG response. 
Pharmacological vs Non-Pharmacological Contributions to the Effects of i.v. NIC

Consistent with our previous work (Kiyatkin and Smirnov, 2010) and early observations in cats (Yamamoto and Domino, 1975), stress- and cue-free injection of pharmacologically neutral saline made during high-magnitude, lowfrequency sleep-like activity rapidly but transiently decreased cortical and VTA EEG total powers, with phasic decreases in $\alpha$ and phasic increases in $\beta$ and $\gamma$ waves (see Figure 2). Although no visual behavioral responses typically occurred following saline injection, the effect on EMG was also significant, but much weaker than that seen with NIC or somato-sensory stimuli. Therefore, this finding suggests that the procedure of i.v. injection per se is a sensory stimulus that is detected by the CNS and induces rapid, transient neural response in both cortex and subcortical structures. While this study did not focus on clarifying the nature of factors responsible for this visceral sensory response, low temperature of injected saline $\left(\sim 23^{\circ} \mathrm{C} v s 37^{\circ} \mathrm{C}\right.$ in the body core $)$ and a transient change in pressure during the injection could be viewed as possible triggering factors. Afferents of visceral sensory nerves abundantly express TRP channels, which are activated by temperature (see Clapham, 2003, for review), and they also contain numerous mechano- and chemoreceptors that detect fluctuations in pressure and ionic properties of blood plasma (Ginzel, 1975).

By analyzing the difference in the effects of NIC and saline (Figure 3), we found that pharmacological contribution to the effects of i.v. NIC on EEG becomes significant at 20-25 s from the injection start, peaks around 1 min post-injection, and decreases slowly afterwards. A similar, even more rapid appearance of a pharmacological effect of NIC was found in the analysis of EMG (5-10 s) and individual EEG waves (10-15s for $\beta$ and $\gamma$ and $15-20 \mathrm{~s}$ for $\alpha$ frequencies). Therefore, although the procedure of i.v. administration contributes to the effects produced by NIC, true pharmacological effects on neural activity and motor outcome become evident within the injection duration (15 s).

\section{Contribution of Sensory Mechanism to the Effects of i.v. NIC: Anesthesia Test}

It is well known that general anesthesia strongly attenuates the central effects of somato-sensory stimuli. If NIC engages a sensory mechanism that is driven from the periphery, it could be expected that the NIC-induced EEG response will be diminished or slowed by anesthesia. Using this approach, we found that rapid effects of i.v. NIC on EEG were markedly reduced in anesthetized conditions, while its motor effects were fully blocked. The immediate effects of NIC during anesthesia were significantly weaker than that of saline, suggesting that general anesthesia also decreases the arousing potential of sensory input from the injection procedure. However, anesthesia did not affect slower effects of NIC, which were equal to those seen in non-anesthesia conditions. Thus, this finding supports the idea that the initial, rapid effects of i.v. NIC are mediated via its interaction with afferents of sensory nerves involving fast neural transmission. In contrast, later-occurring, anesthesiaresistant effects of NIC could be mediated via its direct interaction with centrally located $\mathrm{nACh}$ receptors.
Contributions of Peripheral and Central Actions of NIC to Its Central Effects: The Antagonist and Agonist Tests

If the effects of NIC depend upon its action on peripheral pool of nACh receptors, they should be reduced under conditions when these receptors are blocked. To test for this mechanism, we used HEXA, a peripherally acting blocker of $\mathrm{nACh}$ receptors (antagonist test). Since HEXA fails to cross the $\mathrm{BBB}$, NIC should continue to interact with centrally located nACh receptors, thus revealing its direct central action.

We found that HEXA strongly attenuates NIC-induced EEG desynchronization and EMG activation, significantly decreasing both the rapid and especially long-term changes in both parameters. Therefore, NIC interaction with peripheral nACh receptors appears to provide a major contribution to its central activating effects. Although HEXA also interacts with nACh receptors in neuromuscular junctions and autonomic ganglia, primary afferents of sensory nerves abundantly innervating blood vessels appear to be the primary neural substrates responsible for rapid and strong attenuating effect of this drug when delivered i.v. and in a relatively low dose. Although the effects of NIC during blockade of peripheral nACh receptors were significantly weaker and shorter than in control group, more resembling the effects of saline, HEXA pre-treatment was unable to fully block rapid changes in EEG and EMG, suggesting an additional component of NIC action that is absent in saline. Although it is an effective NIC antagonist, it is difficult to assume that HEXA is able to block all nACh receptors in the ascending pathways that are involved in transmitting the NIC signal from the periphery to the brain at the dose, route of administration, and timing used in this study. Despite these limitations, our results with HEXA could also suggest another intriguing possibility that the peripheral actions of NIC are essential for manifestations of its true pharmacological actions in the brain. Under conditions of peripheral receptor blockade, NIC still produced monophasic changes in both EEG and EMG, and the inhibiting effect of HEXA was evident between 50 and $400 \mathrm{~s}$ post-NIC (see Figure $5 \mathrm{c}$ ), ie, at the time of its presence (Berridge et al, 2010; Rose et al, 2010) and presumed action on central neurons.

To further delineate the contribution of peripheral action of NIC to its central effects, we also employed an agonist strategy, using a peripherally acting NIC analog. This drug is unable to reach centrally located nACh receptors and it has a comparable potency to NIC when assessed in a neuromuscular preparation (Barlow et al, 1969), by pressor effects with i.v. administration (Barlow and Dobson, 1955; Larson and Haig, 1943; Zuo et al, 2009), and in pain tests with central administration (Aceto et al, 1983). Although NIC-PM has been previously used as a peripherally acting NIC agonist (Zuo et al, 2009), and we assumed that it could be the best tool to mimic peripheral actions of NIC, the affinity of this drug to different subtypes of nACh receptors, particularly those located on afferents of sensory nerves, is still unknown.

While NIC-PM induced rapid EEG desynchronization and EMG activation, these effects were much weaker and more transient than those induced by a regular NIC. However, these effects, especially for the first tests with a lower dose, 
were stronger and more prolonged than those induced by saline (see Figure 6; Table 1). In contrast to clear dose dependence and stability of EEG and EMG effects of i.v. NIC following repeated injections, the effects of NIC-PM showed an apparent desensitization, or decrease in strength, with repeated injections. This drug feature could explain an inverted dose dependence, when the effects of first tested NIC-PM at a lower dose were on average stronger than those induced by the same drug at a threefold higher dose but tested later.

However, even taking into consideration a possible inability of NIC-PM to mimic all actions of regular NIC on peripheral $\mathrm{nACh}$ receptors, this agonist test allowed us to confirm and extend the conclusions of the antagonist test. The analysis of differences in the effects of NIC and NIC-PM revealed that the relative contribution of central actions for i.v. NIC becomes evident only at 65 or $100 \mathrm{~s}$ (for cortex and VTA, respectively) after the start of i.v. injection and that this action is very transient (60-80 s). In contrast, NIC-PM and saline comparison revealed that the contribution of peripheral NIC actions (which are presumably mimicked by NIC-PM) is evident immediately after the injection, but generally disappear at $65-70 \mathrm{~s}$ post-injection. This timing agrees with the results obtained in anesthesia and antagonist tests.

\section{Conclusions and Functional Implications}

While the direct action of NIC on central neurons is usually viewed as a basic mechanism underlying its central effects, the results of the present study suggest that interaction of NIC with peripheral neural substrates is the primary factor responsible for its powerful activating effects in the brain. As the NIC sensory signal is rapidly transmitted to the brain from the sites of its primary action (ie, blood vessels, lung alveoli, nasal cavity), these effects occur within second-scale latencies preceding the slower entry of NIC into the brain (Berridge et al, 2010; Rose et al, 1999, 2010) and its direct actions on central neurons. Consistent with this sensory mechanism, the pattern of EEG desynchronization induced by i.v. NIC resembled that seen with sensory stimuli (Kiyatkin and Smirnov, 2010), but the changes had definite onset latencies and were much stronger and more prolonged. Consistent with a well-known inhibiting action of general anesthesia on neural responses to somatosensory stimuli, we found that the initial components of EEG response to i.v. NIC (ie, the change that occurs within the first $60 \mathrm{~s}$ post-injection) were greatly attenuated during urethane anesthesia, without affecting its later effects. Although peripherally acting HEXA was unable to fully block the activating effects of i.v. NIC, they were greatly reduced and shortened. NIC-PM, a peripherally acting NIC analog, was unable to mimic the effects of NIC, but this test revealed a significant contribution of peripheral input within 15-70 s after i.v. injection, corresponding well to the timing $(\sim 60 s)$ of inhibited EEG response in the urethane test. Despite evident proofs for the role of peripheral action of NIC in triggering and maintaining the excitatory effects of NIC, the contribution of its direct actions in the brain, which are the core of its reinforcing properties, remains elusive. However, our tests with NICPM suggest the peripheral actions of NIC alone without subsequent central actions are unable to induce large central effects. Moreover, it appears that the activating effects induced by selective activation of peripheral nACh receptors undergo rapid habituation (desensitization) with repeated stimulation. This contrasts the effects of regular NIC, which remain very stable with repeated use.

If i.v. NIC, by acting on peripheral neural substrates, produces a strong visceral signal that rapidly reaches the brain, central effects produced via this mechanism should always precede the more prolonged direct effects of NIC in the brain. While it is known that NIC could rapidly reach the brain, a definite time is necessary for the peripherally delivered drug to reach cerebral vessels, cross the BBB, and diffuse in brain tissue to its receptive targets (Berridge et al, 2010; Rose et al, 2010). Therefore, similar to the interaction between a sensory stimulus and natural reinforcer that results in conditioning (Pavlov, 1927), two independent pharmacological effects of NIC could interact in the CNS and influence each other following repeated drug use (NIC learning). Similar to indifferent sensory stimuli that gain significance by its Pavlovian association with the primary reinforcer, rapid sensory effects of NIC that are mediated via its interaction with peripheral neural substrates gain its arousing potential with repeated NIC administrations. This basic mechanism could determine both the changes in the behavioral and physiological effects of NIC following its repeated use as well the powerful arousing potential of multiple smoking-related stimuli and cues (ie, smell, sight, taste of smoke/NIC, oro-pharyngeal effects of smoking/NIC) typical of NIC abuse.

\section{ACKNOWLEDGEMENTS}

The research described in this manuscript was supported by the Intramural Research Program of the NIH, NIDA. We thank Drs Charles Spivak, Serge Ahmed, and Alexey Mukhin for valuable comments regarding scientific issues and Jeremy Tang for assistance in editing of this manuscript.

\section{DISCLOSURE}

The authors declare no conflict of interest.

\section{REFERENCES}

Aceto MD, Awaya H, Martin BR, May EL (1983). Antinociceptive action of nicotine and its methiodide derivatives in mice and rats. Br J Pharmacol 79: 869-876.

Alimohammadi H, Silver WL (2000). Evidence for nicotinic acetylcholine receptors on nasal trigeminal nerve endings of the rat. Chem Senses 25: 61-66.

Anand A (1996). Role of aortic chemoreceptors in the hyperthensive response to cigarette smoke. Respir Physiol 106: 231-238.

Balfour DJ (2009). The neuronal pathways mediating the behavioral and addictive properties of nicotine. Handb Exp Pharmacol 192: 209-233.

Barlow RB, Dobson NA (1955). Nicotine monomethiodide. J Pharm Pharmacol 7: 27-34.

Barlow RB, Thompson GM, Scott NC (1969). The affinity and activity of compounds related to nicotine on the rectus abdominis muscle of the frog (Rana pipiens). Br J Pharmacol 37: 555-584. 
Berridge MS, Apana SM, Nagano KK, Berridge CE, Leisure GP, Boswell MV (2010). Smoking produces rapid rise of [11C]nicotine in human brain. Psychopharmacology 209: 383-394.

Buzsaki G (2006). Rhythms of the Brain. Oxford University Press: Oxford.

Byck R (1961). The effect of hexamethonium on the carotid chemoreceptor response to nicotine and cyanide. Brit $J$ Pharmacol Chemother 16: 15-22.

Changeux J-P (2010). Nicotine addiction and nicotinic receptors: lessons from genetically modified mice. Nat Rev Neurosci 11: 389-401.

Clapham DE (2003). TRP channels as cellular sensors. Nature 426: 517-524.

Corrigall WA (1991). Understanding brain mechanisms in nicotine reinforcement. $\mathrm{Br} J$ Addict 86: 507-510.

Cox BM, Goldstein A, Nelson WT (1984). Nicotine self-administration in rats. $\mathrm{Br} J$ Pharmacol 83: 49-55.

Di Chiara G (2000). Role of dopamine in the behavioural actions of nicotine related to addiction. Eur J Pharmacol 393: 295-314.

Donny EC, Caggiula AR, Knopf S, Brown C (1995). Nicotine selfadministration in rats. Psychopharmacology 122: 390-394.

Engberg G, Hajos M (1994). Nicotine-induced activation of locus coeruleus neurons - an analysis of peripheral versus central induction. Naunyn Schmiedeberg's Arch Pharmacol 349: 443-446.

Erhardt S, Schwieler L, Engberg G (2002). Excitatory and inhibitory responses of dopamine neurons in the ventral tegmental area to nicotine. Synapse 43: 227-237.

Flores CM, DeCamp RM, Kilo S, Rogers SW, Hargreaves KM (1996). Neuronal nicotinic receptor expression in sensory neurons of the rat trigeminal ganglion: demonstration of $\alpha 3 \beta 4$, a novel subtype in the mammalian nervous system. $J$ Neurosci 16: 7892-7901.

Gillis CN, Lewis JJ (1956). The pharmacology of nicotine monomethiodide. J Pharm Pharmacol 8: 46-54.

Ginzel KH (1975). The importance of sensory nerve endings as sites of drug action. Naunyn Schmiedeberg's Arch Pharmacol 288: $29-56$.

Gotti C, Clementi F (2004). Neuronal nicotinic receptors: from structure to pathology. Prog Neurobiol 74: 363-396.

$\mathrm{Gu} \mathrm{Q}$, Ni D, Lee LY (2008). Expression of neuronal nicotinic acetylcholine receptors in rat vagal pulmonary sensory neurons. Respir Physiol Neurobiol 161: 87-91.

Hobson JA (1999). Sleep and dreaming. In: Zigmond MJ, Bloom FE, Landis SC, Roberts JL, Squire LR (eds). Fundamental Neuroscience pp 1207-1227, Academic Press: San Diego.

Jonsson M, Kin C, Yamamoto Y, Runold H, Lindahi SG, Eriksson LI (2002). Atracurium and vecuronium block nicotine-induced carotid body chemoreceptor responses. Acta Anaesthesiol Scand 46: 488-494.

Juan H (1982). Nicotinic nociceptors on perivascular sensory nerve endings. Pain 12: 259-264.

Kiyatkin EA, Smirnov MS (2010). Rapid EEG desynchronization and EMG activation induced by intravenous cocaine in freely moving rats: a peripheral, nondopamine neural triggering. $A m \mathrm{~J}$ Physiol 298: R285-R300.

Larson PS, Haig HB (1943). Studies on the fate of nicotine in the body. III. On the pharmacology of some methylated and demethylated derivatives of nicotine. J Pharm Exp Ther 77: 343-349.

Liu L, Simon SA (1996). Capsaicin and nicotine both activate a subset of rat trigeminal ganglion neurons. Am J Physiol 270: C1807-C1814.

McClung R, Reilly E, Dafny N (1976-1977). Urethane modification of EEG-like activity and acoustically evoked field potentials recorded from deep nuclei. Appl Neurophysiol 39: 11-26.

Monti-Bloch L, Eyzaguirre C (1980). A comparative physiological and pharmacological study of cat and rabbit carotid body chemoreceptors. Brain Res 193: 449-470.

Pavlov IP (1927). Conditioned Reflexes (Ed and trans By GV Anrep). Dover: New York.

Paxinos J, Watson C (1998). The Rat Brain in Stereotaxic Coordinates. Academic Press: Sydney.

Rose JE, Behm FM, Westman EC, Coleman RE (1999). Arterial nicotine kinetics during cigarette smoking and intravenous nicotines administration: implications for addiction. Drug Alcohol Depend 56: 99-107.

Rose JE, Mukhin AG, Lokitz SJ, Turkington TG, Herskovic J, Behm FM et al (2010). Kinetics of brain nicotine accumulation in dependent and nondependent smokers assessed with PET and cigarettes containing 11C-nicotine. PNAS 107: 5190-5195.

Sasaki H, Coffey P, Villegas-Perez MP, Vidal-Sanz M, Young MJ, Lund RD et al (1996). Light induced EEG desynchronization and behavioral arousal in rats with restored retinocollicular projection by peripheral nerve graft. Neurosci Lett 218: 45-48.

Steen KH, Reeh PW (1993). Actions of cholinergic agonists and antagonists on sensory nerve endings in rat skin, in vitro. J Neurophysiol 70: 397-405.

Steriade M, McCarley RW (2005). Brain Control of Wakefulness and Sleep. Kluwer Academic/Plenum Publishers: New York.

Toyama T, Yokoyama I, Nishi K (1975). Effects of hexamethonium and other ganglionic blocking agents on electrical activity of the esophagus induced by vagal stimulation in the dog. Eur J Pharmacol 31: 63-71.

Walker JC, Kendal-Reed M, Keiger CJ, Bencherif M, Silver WL (1996). Olfactory and trigemical responses to nicotine. Drug Dev Res 38: 160-168.

Wise RA, Bozarth MA (1987). A psychomotor stimulant theory of addiction. Psychol Rev 94: 469-492.

Xu J, Yang W, Zhang G, Gu Q, Lee LY (2007). Calcium transient evoked by nicotine in isolated rat vagal pulmonary sensory neurons. Am J Physiol 292: L54-L61.

Yamamoto K-I, Domino EF (1975). Nicotine-induced EEG and behavioral arousal. Int J Neuropharmacol 4: 359-373.

Zuo Y, Lu H, Vaupel B, Zhang Y, Yang Y, Stein EA (2009). Neuronal sensitization precedes tolerance to repeated nicotine challenges following overnight withdrawal in chronic nicotinetreated rats. Soc Neurosci Abstr 447: 15.

Supplementary Information accompanies the paper on the Neuropsychopharmacology website (http://www.nature.com/npp) 